\title{
DYNAMIC ARRAY MANIPULATION OF MICROSCOPIC PARTICLES VIA OPTOELECTRONIC TWEEZERS
}

\author{
Aaron T. Ohta, Pei-Yu Chiou, and Ming C. Wu \\ Department of Electrical Engineering, UCLA \\ Los Angeles, CA 90095-1594
}

\begin{abstract}
We report on the first dynamic array manipulation of microparticles using optoelectronic tweezers. Polystyrene particles with 20 and $45-\mu \mathrm{m}$ diameters are individually trapped by light patterns generated by a digital light projector with digital micromirror device (DMD). Self-organization and individual addressing of the particles are demonstrated. Movement of $45-\mu \mathrm{m}$ polystyrene particle is measured to be $35 \mu \mathrm{m} / \mathrm{sec}$ (a force of 15 $\mathrm{pN})$.
\end{abstract}

\section{INTRODUCTION}

Cellular-scale manipulation is an important tool in biological research. Technologies that have demonstrated the capability for such microscopic manipulation include optical tweezers and dielectrophoresis $[1,2]$. While optical tweezers affords very fine control of microparticles, it suffers from high optical power requirements. Dielectrophoresis has been demonstrated to trap particles as small as $14 \mathrm{~nm}$ [3]. However, dielectrophoresis requires a static pattern of electrodes, and is not easily reconfigurable.

We have demonstrated another method of manipulating micrometer-scale objects: optically-induced dielectrophoresis, or optoelectronic tweezers [4]. Using a laser to induce dielectrophoretic forces, we have demonstrated controlled movement of 25- $\mu \mathrm{m}$ latex particles, and E. coli bacteria [4, 5]. This technique allows the use of very low optical power levels, enabling us to perform particle manipulation with an incoherent light source [6]. The use of a spatial light modulator in our optical system also allows us to dynamically reconfigure particle traps, providing us with increased versatility in particle manipulation over conventional dielectrophoresis. In this paper, for the first time, we present dynamic array manipulation of microparticles using optoelectronic tweezers. We demonstrate the selforganization of particles into an array, and the formation of singleparticle arrays, with the capability to individually address each particle.

\section{THEORY}

Dielectrophoresis (DEP) refers to the forces induced upon a particle in the presence of a non-uniform electric field, which are typically generated by a variety of electrode configurations. A particle within an electric field forms an induced dipole, which will experience a force due to the field gradient [2].

The direction of the induced dielectrophoretic force is dependent upon the frequency of the electric field and the permittivity and conductivity of the particle and the surrounding medium. Positive DEP results in particle attraction to electric field maxima. In contrast, negative DEP causes particles to be repelled from field maxima. Applying an ac electric field thus allows the tuning of the type of DEP force induced on a particle, as well as negating any electrophoretic effects, or particle movement due to its surface charge.
Our optoelectronic tweezers (OET) device enables opticallyinduced dielectrophoresis. Unlike conventional DEP, no electrode pattern is required to introduce non-uniformities into an applied electric field; instead, a photoconductive layer is used to form virtual electrodes. Focusing incident light onto the photoconductor greatly increases its conductivity as compared to the dark areas, effectively creating an electrode in the illuminated area, analogous to the patterned electrodes in conventional DEP. In addition, the virtual electrodes used by OET are movable and reconfigurable, unlike the static electrodes of conventional DEP.

To illustrate, a repulsive OET trap is shown in Fig. 1(a). This single-particle rectangular trap has inner dimensions of $70 \mu \mathrm{m}$ by $50 \mu \mathrm{m}$. A sphere with a diameter of $45 \mu \mathrm{m}$ is surrounded by light "walls" $25 \mu \mathrm{m}$ in width. The corresponding cross-sectional distribution of the square of the electric field shows that width of the trap as experienced by the particle is approximately $50 \mu \mathrm{m}$, as DEP force depends on the gradient of this distribution (Fig. 1(b)). If negative DEP forces are induced by the trap pattern, all particles outside the trap area will be repelled by the electric field maxima forming the trap perimeter. Any particle within the enclosed trap area will feel similar repulsive forces, but these forces balance, trapping the particle. Once a particle is contained within the rectangular pattern, the trap can be moved, transporting the particle to a desired location. Furthermore, multiple traps can be used as building blocks to form arrays of trapped particles. Such arrays can be arbitrarily arranged, and dynamically reconfigured.

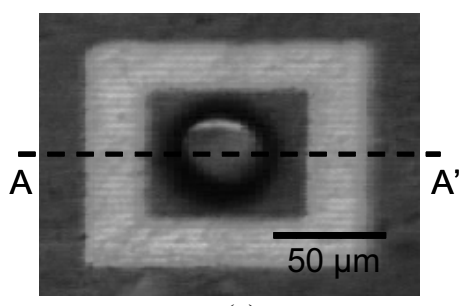

(a)

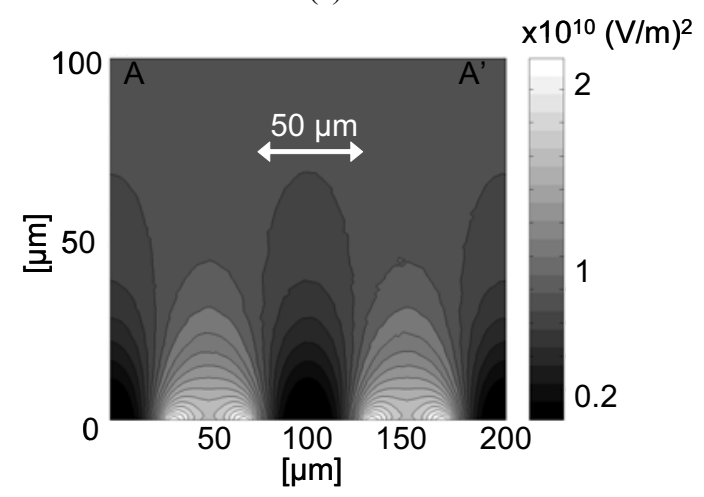

(b)

Figure 1. (a) A single particle OET trap. A $45 \mu$ m polystyrene sphere is contained by optically-induced negative DEP.

(b) Distribution of the square of the electric field for the single particle trap in (a) along the cross-section $A-A$ '. DEP force is proportional to the gradient of this distribution. 
The optical power required to induce DEP forces in OET is much lower than that of optical tweezers, as the light energy does not directly trap the particles. Early experiments using OET showed movement of $25 \mu \mathrm{m}$ particles at $4.5 \mu \mathrm{m} / \mathrm{sec}$ with an optical power of $1 \mu \mathrm{W}$, corresponding to an incident power density of 440 $\mathrm{mW} / \mathrm{cm}^{2}$ [4]. In comparison, a $1 \mu \mathrm{m}$ diameter optical tweezers trap, at a minimum trapping power of $1 \mathrm{~mW}$, has an optical power density of $32 \mathrm{~kW} / \mathrm{cm}^{2}$.

The low optical power requirements of OET offer many advantages in the system design. Inexpensive incoherent light sources can be employed instead of lasers to provide the illumination necessary for OET [6]. In addition, we can produce light patterns by imaging rather than scanning techniques. Furthermore, with no need to focus all optical energy, we can use a simple spatial light modulator to pattern images, rather than the holographic techniques employed by optical tweezers arrays [7]. In our experiments, we used the digital micromirror device (DMD) [8] in a light projector to image the virtual electrodes.

The optoelectronic tweezers device is formed by evaporating a 10-nm-thick aluminum film onto a glass substrate for electrical contact. A $1-\mu \mathrm{m}$-thick undoped amorphous silicon (a-Si) photoconductive layer is then deposited by plasma-enhanced chemical vapor deposition. To protect the photoconductive film, a 20-nm-thick silicon nitride layer is deposited over the a-Si. The liquid buffer layer containing the particles of interest is sandwiched between this photoconductive device and indium-tinoxide (ITO) glass (Fig. 2). An applied ac bias across the ITO and a-Si produces the electric field.

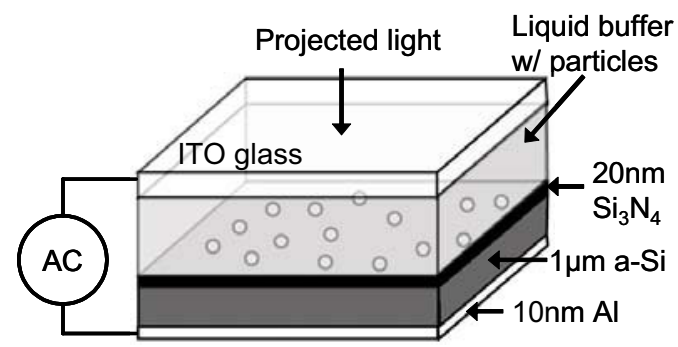

Figure 2. Optoelectronic tweezers device. The liquid buffer containing the particles of interest is contained between the upper ITO glass layer and the lower photoconductive layer. To separate the top and bottom layers, 100- $\mu$ m thick spacers are used (not shown).

Amorphous silicon has a dark conductivity of $0.01 \mu \mathrm{S} / \mathrm{m}$ to 1 $\mu \mathrm{S} / \mathrm{m}$. Thus, in the dark, the a-Si has a much lower conductivity than the liquid buffer (which has a conductivity of $10 \mathrm{mS} / \mathrm{m}$ ), causing the majority of the voltage to drop across the silicon layer. Incident light focused onto the photoconductive layer, greatly increasing its conductivity, results in a non-uniform electric field surrounding the illuminated area, as most of the voltage now drops across the liquid buffer layer. In this manner, the light incident on the OET device can pattern virtual electrodes.

\section{EXPERIMENTAL SETUP}

A DMD-based projector (InFocus LP335) is used to display images drawn on a PC, via Microsoft PowerPoint software. The projector provides both the optical source (a 120W, 1000-ANSI lumen high-pressure mercury lamp) and the DMD-to-PC interface. The output of the projector is collected, collimated, and directed into an Olympus MSPlan10 10X objective lens ( $\mathrm{NA}=0.30$ ), projecting an image onto the OET device. The power at the projector output was measured to be approximately $600 \mathrm{~mW}$.
Approximately $7 \%$ of this power is collected by the objective lens and focused onto the OET device. Therefore, the power of the light incident on the OET is $42 \mathrm{~mW}$, corresponding to an intensity of $12 \mathrm{~W} / \mathrm{cm}^{2}$.

The buffer solution consists of deionized water and KCL salt, mixed to obtain a conductivity of $10 \mathrm{mS} / \mathrm{m}$. Polystyrene microspheres (45 and $20 \mu \mathrm{m}$, from Polysciences, Inc.) are mixed into the buffer solution, and sandwiched into the OET device. Observation of the particles under test is done via a Nikon TE2000U inverted microscope. A CCD camera attached to the observation port of the microscope recorded images and video of our experiments. A schematic of the optical setup is shown in Fig. 3. To produce the electric field necessary for DEP, an ac voltage of 10Vpp at $100 \mathrm{kHz}$ (Agilent 33120A) was applied across the top ITO surface and the bottom photoconductive surface of the OET device.

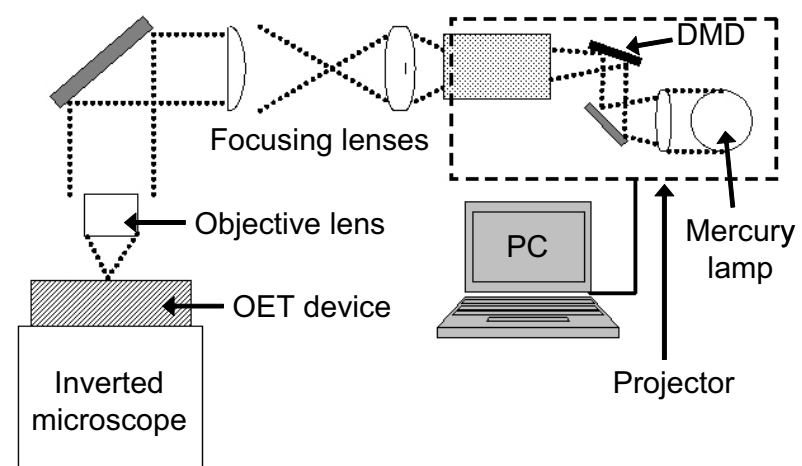

Figure 3. Experimental setup. The image from an InFocus LP335 DMD-based projector is focused into a $10 x$ objective lens, and projected down onto the OET device. Particle movement is observed via the Nikon TE2000U inverted microscope.

\section{EXPERIMENTAL RESULTS}

We have demonstrated the self-organization of randomly distributed $45-\mu \mathrm{m}$ polystyrene spheres into an array (Fig. 4). A simple grid pattern consisting of orthogonal horizontal and vertical lines, drawn in PowerPoint, is then projected onto the OET device. The pattern activates the optically-induced DEP, repelling particles from the illuminated areas due to negative DEP forces. This mechanism causes the self-organization of the particles once the grid pattern is illuminated; the particles are pushed into the nonilluminated cells. After a settling period, the particles are trapped within the of an array cells.

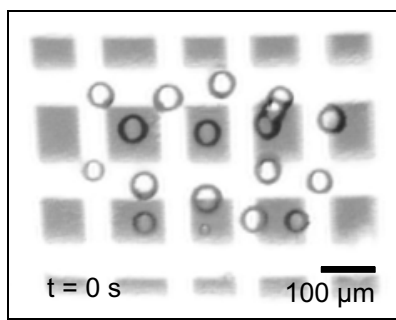

(a)

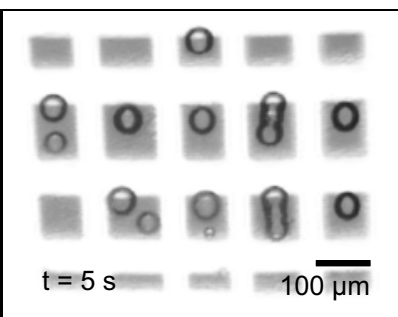

(b)

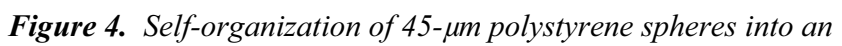
array configuration. After the initial grid illumination (a), the randomly arranged particles move towards the dark areas via negative DEP. After five seconds, all particles are contained within the array cells (b). 
Due to a large trap relative to the particle size, the initial selforganization may result in more than one particle per array cell (Fig. 4(b)). In this array, the largest cells are $80 \mu \mathrm{m}$ by $100 \mu \mathrm{m}$. It may be possible to form self-organizing arrays with a single particle per array cell by optimizing the dimensions of a single array cell trap, such that only one particle may fit into the trap's potential well at any time.

In addition, we found that certain particles within the selforganized array are able to escape when the array is moved around the image plane. This phenomenon occurs for the array cells that contain multiple particles. This occurrence, along with subsequent manipulation of the self-organized array in Fig. 4(b), allowed us to obtain an array with a single particle per cell (Fig. 5a). We were able to move the resulting array of single particles around the image plane at approximately $25 \mu \mathrm{m} / \mathrm{sec}$.

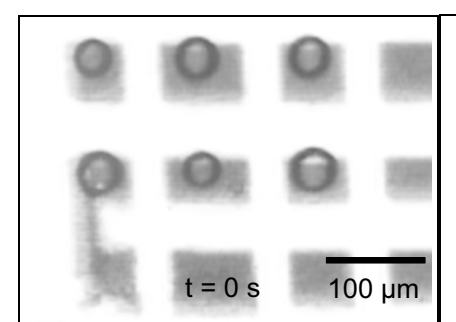

(a)

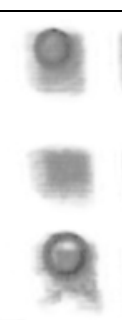

(b)
Figure 5. Single-particle manipulation within the array. A particle in the lower-left side of the array is made to change its array by combining cells (a), the re-splitting the cell, moving the particle to the adjacent array position (b).

Particles can be moved individually between adjacent cells, as illustrated in Fig. 5. The adjacent cells are merged by first removing the dividing wall, and then re-separating the cells. All movement of the trap walls are controlled in real time by the operator. To improve on the speed of this technique, a moving light wall can be used to facilitate the transportation of the particle between cells. This enables a single particle to be transferred to any cell of the array, using repeated transfers between adjacent cells.

Since the patterns for manipulating the particles in the array are created dynamically by optical illumination, a wide variety of operations can be performed by simple software programming. For example, to flush the particles in a single row of the array, we remove the dividing walls of that row and use a moving wall to sweep out the particles (Fig. 6).

In addition to self-organizing behavior, arrays can be formed from multiple single-particle traps. Each randomly positioned particle is first contained within a square trap. This is performed by drawing a rectangle around each particle in PowerPoint. The multiple traps can then be positioned to form an array of individually addressable cells. Using this technique, we are able to form a $4 \times 5$ array of single particle traps (Fig. 7). Though the operation was performed manually, it can potentially be automated by combining OET with a vision system. Biological applications of such an array include studies on single-cell behavior and interaction.

Since each cell of the array is an independent single particle trap, the array has the capability of being dynamically rearranged. To illustrate, an array consisting of $45 \mu \mathrm{m}$ and $20 \mu \mathrm{m}$ polystyrene spheres are reorganized under operator control (Fig. 8). This demonstrates the addressability of each particle trap, as well as the dynamic nature of the OET patterns.

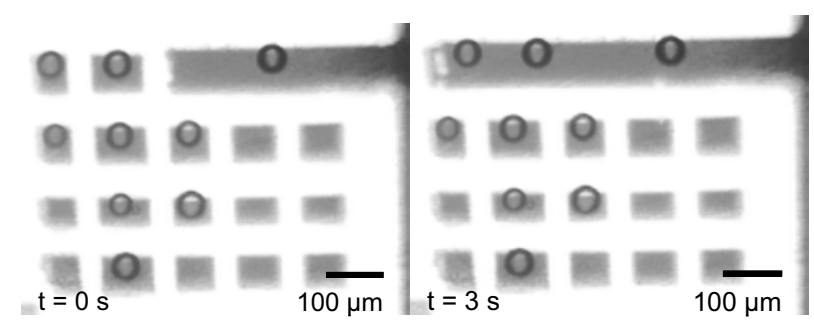

(a)

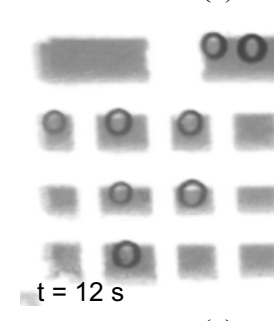

(c) (b)

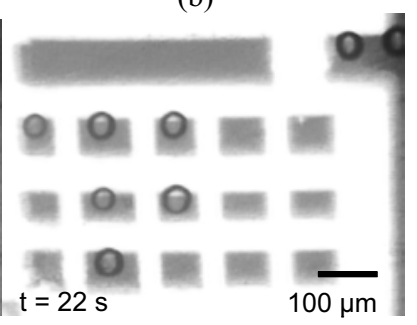

(d)
Figure 6. Flushing an array row (to remove undesired particles from the array). First, the walls of the cells in the row to be flushed are removed (top row in (a)). The particles are no longer bounded in the lateral direction (b). An operator-controlled light bar is then used to push the particles out of the array $(c, d)$.

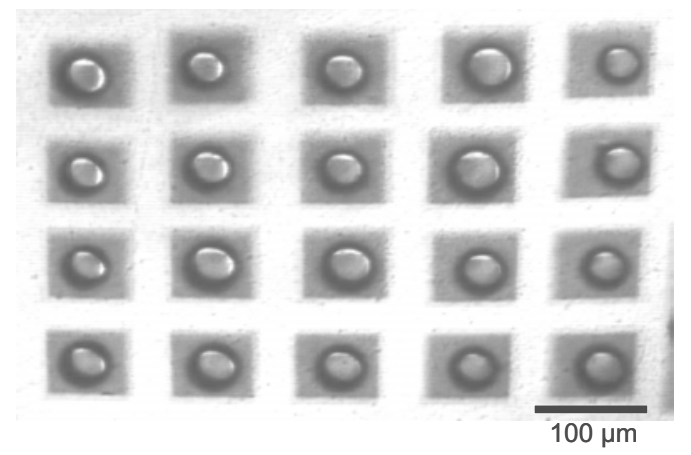

Figure 7. An array of single particles, formed from multiple single particle square traps. Each particle is individually addressable. The time required to form this array of 20 particles was 3 minutes.

\section{DISCUSSION}

Movement of a single $45-\mu \mathrm{m}$ sphere due to negative DEP has a maximum velocity of approximately $35 \mu \mathrm{m} / \mathrm{sec}$. This corresponds to an estimated force of $15 \mathrm{pN}$, based upon Stoke's Law. The maximum velocity of a 20-particle array is limited to approximately $25 \mu \mathrm{m} / \mathrm{sec}$. Thus, the minimum holding force of each individual array cell is $10 \mathrm{pN}$. This force is less than that experienced by a single $45 \mu \mathrm{m}$ particle, probably due to slight nonuniformities in image sharpness over the entire array area. The more defocused areas will have less of an electric field gradient, and a correspondingly lower DEP force. Thus, this $10 \mathrm{pN}$ force reflects the minimum trapping force of all of the array cells.

The forces attained in these experiments, using an optical power density of $12 \mathrm{~W} / \mathrm{cm}^{2}$, are in rough agreement with our earlier results using a 632-nm laser light source [4]. Our earlier data suggests that the optical power density necessary to achieve a force of $15 \mathrm{pN}$ is $6.6 \mathrm{~W} / \mathrm{cm}^{2}$. The difference between this predicted power density requirement and our experimental findings can be attributed to losses through the additional optics needed for our current experiment. 


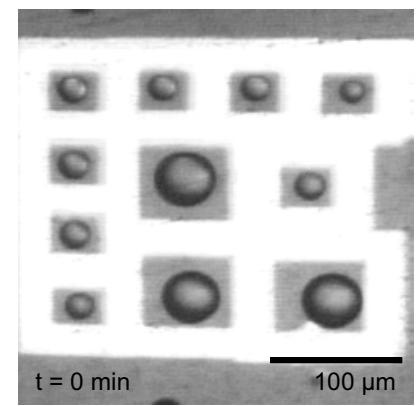

(a)

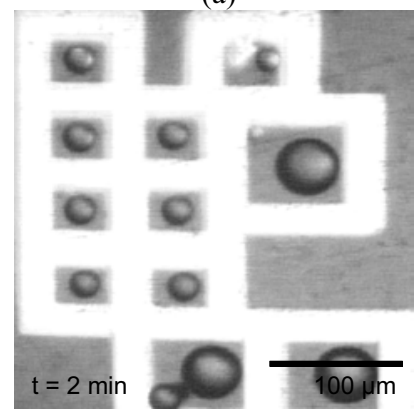

(c)
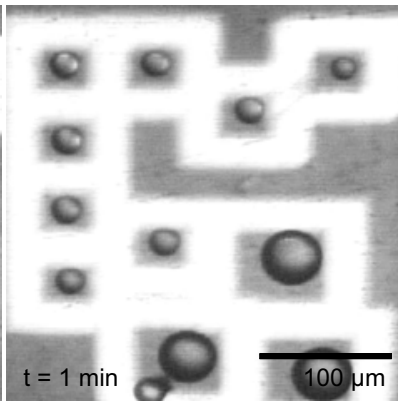

(b)

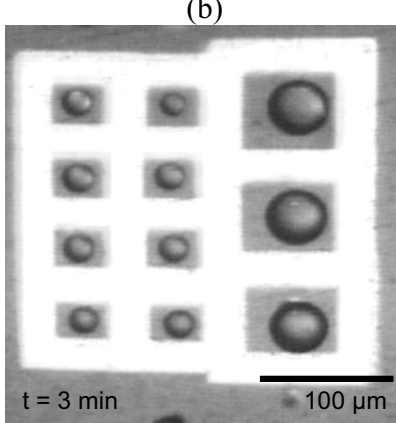

(d)
Figure 8. Dynamic rearrangement of an array containing both 45 $\mu \mathrm{m}$ and $20 \mu \mathrm{m}$ particles. An array is rearranged by moving individual cells into a desired configuration. Total rearrangement time is 3 minutes.

Our results compare favorably to other microparticle manipulation techniques. Conventional dielectrophoresis uses static electrode patterns, and is thus not reconfigurable. In addition, our device is less expensive to produce, as no photolithographic steps are needed. Addressable DEP arrays have also been demonstrated using CMOS technology [9], but these devices are expensive to produce, and the minimum electrode size is limited by the required CMOS circuits $(20 \mu \mathrm{m}$ in [9]). Both conventional DEP and optical tweezers are capable of manipulating particles a few nanometers in diameter. The minimum size of the virtual electrode in OET is limited by the 115-nm ambipolar diffusion length of the a-Si [10].

The OET can operate over a large area $\left(\sim 1 \times 1 \mathrm{~mm}^{2}\right)$, which is much greater than the $20 \times 20 \mu \mathrm{m}^{2}$ for optical tweezers [7]. Though holographic tweezers can generate multiple traps, direct imaging using a DMD is more versatile. It can generate any arbitrary pattern with high contrast ratio. No computation is required to generate the desired pattern. Furthermore, OET can induce repulsive forces on transparent dielectric particles such as biological cells, and form cell cages, which is not possible with optical tweezers. On the other hand, optical tweezers traps are three-dimensional, whereas our trap patterns are limited to two dimensions. We are also must be more selective in our choice of buffer solutions, as the conductivity of the solution plays a crucial role in the DEP phenomenon.

\section{CONCLUSIONS}

We have demonstrated the self-organizing of 45-um polystyrene particles into an array, and the creation of an array from multiple single particle traps utilizing optoelectronics tweezers. Single particle movement within the array has been demonstrated, showing the ability to address individual array cells.
Movement of single $45 \mu \mathrm{m}$ polystyrene spheres was measured to be $35 \mu \mathrm{m} / \mathrm{sec}$ (a force of $15 \mathrm{pN}$ ). Movement of a 20-particle array was performed at $25 \mu \mathrm{m} / \mathrm{sec}$ (a force of $10 \mathrm{pN}$ ). Such particle manipulation techniques have many applications to experiments with biological cells and microparticles.

\section{ACKNOWLEDGEMENTS}

This project is supported by the Center for Cell Mimetic Space Exploration (CMISE), a NASA University Research, Engineering, and Technology Institute (URETI). Travel support has been generously provided by the Transducers Research Foundation and by the DARPA MEMS and BioFlips programs.

\section{REFERENCES}

[1] A. Ashkin, J. M. Dziedzic, J. E. Bjorkholm, and S. Chu, "Observation of a single-beam gradient force optical trap for dielectric particles," Optics Letters, 11(5), pp. 288-290, 1986.

[2] H. A. Pohl, Dielectrophoresis. Cambridge: Cambridge University Press, 1978.

[3] T. Muller, A. Gerardino, T. Schnelle, S. G. Shirley, F. Bordoni, G. Degasperis, R. Leoni, and G. Fuhr, "Trapping of micrometer and sub-micrometer particles by high-frequency electric fields and hydrodynamic forces," J. Physics D - Applied Physics, 29(2), pp. 340-349, 1996.

[4] P. Y. Chiou, Z. Chang, Zehao, and M. C. Wu, "A Novel Optoelectronic Tweezer Using Light Induced Dielectrophoresis," Proc. IEEE/LEOS International Conf. Optical MEMS, pp. 8-9, 2003.

[5] P. Y. Chiou, W. Wong, J. C. Liao, and M. C. Wu, "Cell Addressing and Trapping Using Novel Optoelectronic Tweezers," Proc. IEEE MEMS, pp. 21-24, 2004.

[6] A. T. Ohta, P. Y. Chiou, and M. C. Wu, "Dynamic DMDDriven Optoelectronics Tweezers for Microscopic Particle Manipulation," to be published in the Proc. Conference on Lasers and Electro Optics (CLEO) 2004.

[7] D. G. Grier, "A revolution in optical manipulation," Nature, vol. 424, pp. 810-816, 2003.

[8] L. J. Hornbeck, "Deformable-Mirror Spatial Light Modulators," Spatial Light Modulators and Applications III, SPIE Critical Reviews, vol. 1150, pp. 86-102, 1989.

[9] N. Manaresi, A. Romani, G. Medoro, L. Altomare, A. Leonardi, M. Tartagni, R. Guerrieri, "A CMOS Chip for Individual Cell Manipulation and Detection," Proceedings of IEEE International Solid-State Circuits Conference 2003, vol. 1, pp. 192-487, 2003.

[10] R. Schwarz, F. Wang, M. Reissner. "Fermi-level dependence of the ambipolar diffusion length in amorphous silicon thin film transistors," Applied Physics Letters, vol. 63, p.1083, 1993. 